\title{
Application of Extrapolation Methods to Numerical Solution of Fredholm Integral Equations Related to Boundary Value Problems
}

\author{
Avram Sidi \\ Computer Science Department, Technion - Israel Institute of Technology, \\ Haifa 32000, Israel \\ asidi@cs.technion.ac.il
}

\begin{abstract}
Fredholm integral equations arise naturally in the context of ordinary and partial differential equations: Two-point boundary value problems can be reformulated as Fredholm integral equations, whose kernels are continuous but have finite jump discontinuities in their derivatives. Two-dimensional elliptic boundary problems can be reformulated as Fredholm integral equations with kernels that have singularities, some having logarithmic singularities. In this note, we describe quadrature methods whose accuracies can be improved at will. These are obtained by improving the underlying numerical quadrature formulas in a clever fashion. In the case of two-point boundary value problems, they are obtained by correcting the trapezoidal rule appropriately to the accuracy required. In the case of boundary integral equations, they are obtained by first correcting the basic trapezoidal rule and then extrapolating it to required accuracy.
\end{abstract}

\section{Introduction}

In this note, we describe two quadrature methods for the numerical solution of Fredholm integral equations that have been proposed recently. Despite the singular nature of the integral equations involved, these methods turn out to be very efficient in the sense that their accuracies can be improved at will, the increase in their computational costs being minor.

The relevant integral equations are those that arise in the context of ordinary and partial differential equations with boundary conditions. We give two examples of such problems now.

1. Let the two-point boundary value problem

$$
y^{\prime \prime}=f(x, y), \quad 0<x<1 ; \quad a_{0} y(0)+b_{0} y^{\prime}(0)=c_{0}, \quad a_{1} y(1)+b_{1} y^{\prime}(1)=c_{1},
$$

be given. Here $f(x, y)$ is a linear or nonlinear function in $y$. Subtracting $k^{2} y$, with $k>0$ chosen appropriately (even "optimally"), from both sides of the differential equations, and using Green's functions, this problem can be reformulated in the form

$$
y(x)=r(x)+\int_{0}^{1} g(x, t)\left[k^{2} y(t)-f(t, y(t))\right] d t,
$$


with appropriate $r(x)$ and $g(x, t)$. Here the Green's functions are continuous for $x=t$ but their partial derivatives have finite jump discontinuities across $x=t$; they are smooth everywhere else on $[0,1] \times[0,1]$. An important advantage of approximating $y(x)$ via the integral equation formulation is that, in case of singularly perturbed problems, boundary layers, if present, can be resolved with great precision. Recall that this is a difficult task when the differential equation is attacked with difference methods.

For example, with $y^{\prime}(0)=0$ and $y(1)=1$ as boundary conditions, $r(x)$ and $g(x, t)$ are given by

$$
r(x)=\frac{\cosh k x}{\sinh k x}, \quad g(x, t)=\frac{1}{k \cosh k}\left\{\begin{array}{l}
\cosh k x \sinh k(1-t), \quad 0 \leq x \leq t \\
\sinh k(1-x) \cosh k t, \quad t \leq x \leq 1
\end{array} .\right.
$$

This approach was initiated by Keller [1, who used it to prove constructive existence and uniqueness theorems for solutions of the nonlinear problems above and also for designing a quadrature method based on the trapezoidal rule for computing numerical solutions. It was refined and developed further by Pennline [2], [3], 4].

2. Let $u(x, y)$ be the solution of the two-dimensional Laplace's equation with Dirichlet boundary condition

$$
\Delta u(P)=0, \quad P \in \Omega ; \quad u(P)=f(P) \quad P \in \partial \Omega,
$$

where $\Delta$ is the two-dimensional Laplacian, $\Delta=\partial^{2} / \partial x^{2}+\partial^{2} / \partial y^{2}, \Omega$ is a finite simply connected open domain in the $x$ - $y$ plane, and $\partial \Omega$ is its boundary. $u(x, y)$ can be obtained from the integral

$$
u(P)=\int_{\partial \Omega} \rho(Q) \log |P-Q| d l_{Q}, \quad P \in \Omega,
$$

where $d l_{Q}$ is the line element on $\partial \Omega$, and $\rho(Q)$ is the solution of the Fredholm integral equation of the first kind

$$
\int_{\partial \Omega} \rho(Q) \log |P-Q| d l_{Q}=f(P), \quad P \in \partial \Omega .
$$

This is the so-called boundary integral equation formulation of the interior Dirichlet problem. A nice feature of this formulation is that its dimension is one less than that of the original differential equation formulation.

Now, the kernel $g(P, Q)=\log |P-Q|$ of the new formulation is smooth for all $Q \in \partial \Omega$, except when $Q=P$, where it has a logarithmic singularity. This is easily seen after parameterizing the closed curve $\partial \Omega$ appropriately. Also, in terms of the parameter used to represent $\partial \Omega, g(P, Q)$ is periodic in $P$ and $Q$, and the solution $\rho(P)$ and the right-hand side $f(P)$ are periodic in $P$. In addition, if $\partial \Omega$ is smooth, then $\rho(P), P \in \partial \Omega$, is also a smooth function. These facts can be used to advantage when treating the integral equation numerically. 


\section{Treatment of Kernels with Jump Discontinuities}

We begin with integral equations of the form

$$
y(x)=\int_{0}^{1} g(x, t) F(t, y(t)) d t, \quad 0 \leq x \leq 1,
$$

where (i) $r(x)$ is continuous on $[0,1]$, (ii) $g(x, t)$ is continuous over the set $[0,1] \times$ $[0,1]$, its partial derivatives $g_{k}(x, t) \equiv \frac{\partial^{k}}{\partial t^{k}} g(x, t), k=1,2, \ldots$, are smooth for $0 \leq t \leq x$ and for $x \leq t \leq 1$, with finite jump discontinuities across $t=x$, namely, $g_{k}(x, x+)-g_{k}(x, x-)=\delta_{k}(x) \in C[0,1], k=1,2, \ldots$, and (iii) $F(x, y)$ is linear or nonlinear in $y$ and smooth on some set $\Delta=[0,1] \times\left[R_{1}, R_{2}\right]$, where $\left[R_{1}, R_{2}\right]$ is a finite, semi-infinite, or infinite interval.

The numerical treatment of this integral equation has been considered recently by Sidi and Pennline [8], and we follow this treatment here.

If a solution to (11) exists, then it can be shown that it is smooth over $[0,1]$. We assume that a unique solution $y(x)$ exists and that $R_{1} \leq y(x) \leq R_{2}$ when $0 \leq x \leq 1$. A sufficient condition for this to be true is

$$
\left[\max _{x \in[0,1]} \int_{0}^{1}|g(x, t)| d t\right]\left[\max _{(x, w) \in \Delta}\left\|\frac{\partial}{\partial w} F(x, w)\right\|\right]<1 .
$$

Choose a positive integer $N$ and set $h=1 / N$ and $x_{i}=i h, i=0,1, \ldots, N$. Next, for $x=x_{i}, i \in\{0,1, \ldots, N\}$, we approximate the integral

$$
I(x)=\int_{0}^{1} \phi(t) d t, \quad \phi(t)=g(x, t) F(t, y(t)),
$$

via the standard trapezoidal rule

$$
T(h)=h \sum_{j=0}^{N} \prime \prime \phi\left(x_{j}\right), \quad \sum_{j=0}^{N}{ }^{\prime \prime} \alpha_{j}=\frac{1}{2} \alpha_{0}+\sum_{i=1}^{N-1} \alpha_{j}+\frac{1}{2} \alpha_{N} .
$$

Now, by the fact that $F(t, y)$ is smooth in $[0,1] \times\left[R_{1}, R_{2}\right]$ and by the assumed properties of $g(x, t), \phi(t)$ is smooth for $t \in[0, x]$ and $t \in[x, 1]$, continuous for $t \in[0,1]$ with finite jump discontinuities across $t=x$ in all its derivatives. Consequently, for $x=x_{i} \in(0,1)$, i.e., for $i \in\{1,2, \ldots, N-1\}, T(h)$ has the following Euler-Maclaurin expansion:

$$
\begin{aligned}
I(x)=T(h) & -\sum_{s=1}^{p-1} \frac{B_{2 s}}{(2 s) !}\left[\phi^{(2 s-1)}(1)-\phi^{(2 s-1)}(0)\right] h^{2 s} \\
& +\sum_{s=1}^{p-1} \frac{B_{2 s}}{(2 s) !}\left[\phi^{(2 s-1)}(x+)-\phi^{(2 s-1)}(x-)\right] h^{2 s}+E(h),
\end{aligned}
$$

where $E(h)=O\left(h^{2 p}\right)$ as $h \rightarrow 0$, and uniformly in $i$. For $x=x_{0}=0$ and $x=x_{N}=$ 1 , the summation involving the terms containing $\left[\phi^{(2 s-1)}(x+)-\phi^{(2 s-1)}(x-)\right]$ is absent from (2). 
Obviously, $I(x)-T(h)=O\left(h^{2}\right)$ as $h \rightarrow 0$, and thus the accuracy of $T(h)$ is simply too low. The formula

$$
\begin{aligned}
T_{p}(h)=T(h) & -\sum_{s=1}^{p-1} \frac{B_{2 s}}{(2 s) !}\left[\phi^{(2 s-1)}(1)-\phi^{(2 s-1)}(0)\right] h^{2 s} \\
& +\sum_{s=1}^{p-1} \frac{B_{2 s}}{(2 s) !}\left[\phi^{(2 s-1)}(x+)-\phi^{(2 s-1)}(x-)\right] h^{2 s},
\end{aligned}
$$

on the other hand, satisfies $I(x)-T_{p}(h)=O\left(h^{2 p}\right)$ as $h \rightarrow 0$. Since $T_{p}(h)$ involves derivatives of the unknown function $y(x)$, we cannot make direct use of it, unfortunately. However, we can approximate $T_{p}(h)$ via another formula $\hat{T}_{p}(h)$, involving only the values of $y(x)$ and no derivatives of it, for which $I(x)-\hat{T}_{p}(h)=O\left(h^{2 p}\right)$ as $h \rightarrow 0$ as well.

We show how this is done only for $p=2$, and refer the the reader to [8] for all other values of $p$. We have, for $x=x_{i}, i \in\{1,2, \ldots, N-1\}$,

$$
T_{2}(h)=T(h)-\frac{B_{2}}{2}\left\{\left[\phi^{\prime}(1)-\phi^{\prime}(0)\right]-\left[\phi^{\prime}(x+)-\phi^{\prime}(x-)\right]\right\} h^{2} .
$$

We first break up $\phi^{\prime}(t)$ in the form

$$
\phi^{\prime}(t)=g_{1}(x, t) F(t, y(t))+g(x, t) \frac{d}{d t} F(t, y(t)) .
$$

Then we approximate $\phi^{\prime}(0)$ and $\phi^{\prime}(1)$ by using one-sided numerical differentiation formulas for $\frac{d}{d t} F(t, y(t))$ at $t=0$ and $t=1$. These are

$$
\begin{gathered}
Q^{\prime}(0)=\frac{1}{2 h}[-3 Q(0)+4 Q(h)-Q(2 h)]+\frac{1}{3} Q^{\prime \prime \prime}(\xi) h^{2}, \quad 0<\xi<2 h, \\
Q^{\prime}(1)=\frac{1}{2 h}[3 Q(1)-4 Q(1-h)+Q(1-2 h)]+\frac{1}{3} Q^{\prime \prime \prime}(\xi) h^{2}, \quad 1-2 h<\xi<1 .
\end{gathered}
$$

Next, by continuity of $g(x, t)$ across $t=x$, we have

$$
\phi^{\prime}(x+)-\phi^{\prime}(x-)=\left[g_{1}(x, x+)-\left[g_{1}(x, x-)\right] F(x, y(x))=\delta_{1}(x) F(x, y(x)) .\right.
$$

$\hat{T}_{2}(h)$ is obtained by combining these in $T_{2}(h)$, and we obviously have $I(x)-$ $\hat{T}_{2}(h)=O\left(h^{4}\right)$ as $h \rightarrow 0$. We finally use $\hat{T}_{2}(h)$ to define our quadrature method for the integral equation (1).

The resulting quadrature method is defined via the following $N+1$ equations:

$$
\begin{aligned}
y_{i}=r\left(x_{i}\right) & +h \sum_{j=0}^{N} \prime \prime g\left(x_{i}, x_{j}\right) F_{j} \\
& -\frac{h}{24}\left[g\left(x_{i}, 1\right)\left(3 F_{N}-4 F_{N-1}+F_{N-2}\right)-g\left(x_{i}, 0\right)\left(-3 F_{0}+4 F_{1}-F_{2}\right)\right] \\
& -\frac{h^{2}}{12}\left[g_{1}\left(x_{i}, 1\right) F_{N}-g_{1}\left(x_{i}, 0\right) F_{0}\right]+\frac{h^{2}}{12} \delta_{1}\left(x_{i}\right) F_{i}, \quad i=1,2, \ldots, N-1,
\end{aligned}
$$




$$
\begin{aligned}
y_{0}=r\left(x_{0}\right) & +h \sum_{j=0}^{N} \prime \prime g\left(x_{0}, x_{j}\right) F_{j} \\
& -\frac{h}{24}\left[g(0,1)\left(3 F_{N}-4 F_{N-1}+F_{N-2}\right)-g(0,0)\left(-3 F_{0}+4 F_{1}-F_{2}\right)\right] \\
& -\frac{h^{2}}{12}\left[g_{1}(0,1) F_{N}-g_{1}(0,0+) F_{0}\right] \\
y_{N}=r\left(x_{N}\right) & +h \sum_{j=0}^{N} \prime g\left(x_{N}, x_{j}\right) F_{j} \\
& -\frac{h}{24}\left[g(1,1)\left(3 F_{N}-4 F_{N-1}+F_{N-2}\right)-g(1,0)\left(-3 F_{0}+4 F_{1}-F_{2}\right)\right] \\
& -\frac{h^{2}}{12}\left[g_{1}(1,1-) F_{N}-g_{1}(1,0) F_{0}\right] .
\end{aligned}
$$

Here $F_{i} \equiv F\left(x_{i}, y_{i}\right)$ and $y_{i}$ is the approximation to $y\left(x_{i}\right)$.

Using precisely the same procedure, we can derive a quadrature method for (1) that is based on the quadrature formula $\hat{T}_{p}(h)$. Again, this method involves the numerical solution of $N+1$ equations in the unknowns $y_{j}$. As shown in [8], under appropriate conditions, this system has a unique solution for the $y_{j}$ when $N$ is sufficiently large, and that $y\left(x_{j}\right)-y_{j}=O\left(h^{2 p}\right)$ as $h \rightarrow 0$ (equivalently, $N \rightarrow \infty)$, this error being uniform in $j$.

The quadrature method based on $\hat{T}_{2}(h)$ is illustrated with two examples involving nonlinear two-point boundary value problems in [8].

\section{Treatment of Kernels with Logarithmic Singularity}

We now turn to the numerical solution of Fredholm integral equations of the form

$$
\omega f(t)+\int_{a}^{b} K(t, x) f(x) d x=g(t), \quad a \leq t \leq b ; \quad \omega=0,1 .
$$

(Such equations are of the first or the second kind depending on whether $\omega=0$ or $\omega=1$, respectively.) The equations that are of interest to us here have the following important features: (i) The kernel $K(t, x)$ is periodic both in $t$ and in $x$ with period $T=b-a$ and is infinitely differentiable in $(-\infty, \infty) \backslash\{t+k T\}_{k=-\infty}^{\infty}$. It has a polar singularity and/or a branch singularity (of algebraic or logarithmic or algebraic-logarithmic type) at $x=t$ when $t \in[a, b]$. (ii) The input function $g(t)$ and the solution $f(t)$ are both periodic with period $T=b-a$ and infinitely smooth on $(-\infty, \infty)$.

In case $K(t, x)$ has an integrable singularity across $x=t$, (4) is said to be weakly singular. In case $K(t, x) \sim c /(x-t)$ as $x \rightarrow t$ for some constant $c \neq 0$, and the integral $\int_{a}^{b} K(t, x) f(x) d x$ is defined only in the Cauchy principal value sense, it is said to be singular. 
The numerical treatment of these integral equations has been considered recently by Sidi and Israeli [7. A comparison of the quadrature formulas proposed there with others has been given in 5]. For an extensive summary of the subject, see Sidi [6, Section 25.3 and Appendix D]. Here we restrict our attention to weakly singular integral equations with only logarithmic singularities across $x=t$. For other types of singularities, the reader is referred to [7] and [6].

A logarithmically singular kernel can be expressed in the form

$$
K(t, x)=H_{1}(t, x) \log |t-x|+H_{2}(t, x),
$$

where $H_{1}(t, x)$ and $H_{2}(t, x)$ are infinitely differentiable for all $t, x \in[a, b]$ (including $t=x)$, but are not necessarily periodic. We assume that $H_{1}(t, t) \not \equiv 0$. Note that, in our method below, we do not require $H_{1}(t, x)$ and $H_{2}(t, x)$ for all $x$, but only for $x=t ; H_{1}(t, t)$ and $H_{2}(t, t)$ can be obtained simply by expanding $K(t, x)$ about $x=t$.

Let $n$ be a positive integer and set $h=T / n$ and $x_{i}=a+i h, i=1, \ldots, n$. In addition, let $t \in\left\{x_{1}, \ldots, x_{n}\right\}$. Let us now define the "corrected" trapezoidal rule approximations $I[h ; t]$ to the integral $I[t]=\int_{a}^{b} K(t, x) f(x) d x$ as in

$$
I[h ; t]=\sum_{i=1}^{n} w_{n}\left(t, x_{i}\right) f\left(x_{i}\right)
$$

where

$$
w_{n}(t, x)=h K(t, x) \text { for } x \neq t, \quad w_{n}(t, t)=h\left[H_{2}(t, t)+H_{1}(t, t) \log \left(\frac{h}{2 \pi}\right)\right] .
$$

$I[h ; t]$ has the asymptotic (Euler-Maclaurin) expansion

$$
I[h ; t] \sim I[t]+\sum_{k=1}^{\infty} \beta_{k}(t) h^{2 k+1} \quad \text { as } h \rightarrow 0,
$$

where

$$
\beta_{k}(t)=-\left.2 \frac{\zeta^{\prime}(-2 k)}{(2 k) !} \frac{\partial^{2 k}}{\partial x^{2 k}}\left[H_{1}(t, x) f(x)\right]\right|_{x=t}, \quad k=1,2, \ldots
$$

Here $\zeta(s)$ is the Riemann Zeta function. From these expansions, it follows that $I[h ; t]-I[t]=O\left(h^{3}\right)$ as $h \rightarrow 0$.

The quadrature method based on the rule $I[h ; t]$ is now defined by the equations

$$
\omega \tilde{f}_{k}+I\left[h ; x_{k}\right]=g\left(x_{k}\right), \quad k=1,2, \ldots, n .
$$

More explicitly, these equations are

$$
\omega \tilde{f}_{k}+\sum_{i=1}^{n} w_{n}\left(x_{k}, x_{i}\right) \tilde{f}_{i}=g\left(x_{k}\right), \quad k=1,2, \ldots, n,
$$


where $\tilde{f}_{i}$ is the approximation to $f\left(x_{i}\right)$. In general, the accuracy of the $\tilde{f}_{i}$ is the same as that of the underlying numerical quadrature formula, which is $I[h ; t]$ in this case. We can increase the accuracy of the quadrature method by increasing that of $I[h ; t]$, which we propose to achieve by using extrapolation. What makes this possible is the periodicity of the integrand $K(t, x) f(x)$ as a function of $x$. We turn to this subject next.

We start by using only one extrapolation to eliminate the term $\beta_{1}(t) h^{3}$ from the asymptotic expansion of $I[h ; t]$. Let us choose $h=T / n$ for some even integer $n$ and let $x_{i}=a+i h, i=0,1, \ldots, n$. Performing this single extrapolation, we obtain the Romberg-type quadrature rule

$$
J_{1}[h ; t]=\frac{8}{7} I[h ; t]-\frac{1}{7} I[2 h ; t]
$$

as the new approximation to $I[t]$. We also have

$$
J_{1}[h ; t] \sim I[t]+\sum_{k=2}^{\infty} \frac{2^{3}-2^{2 k+1}}{7} \beta_{k}(t) h^{2 k+1} \text { as } h \rightarrow 0,
$$

hence $J_{1}[h ; t]-I[t]=O\left(h^{5}\right)$ as $h \rightarrow 0$. The quadrature method for (4) based on $J_{1}[h ; t]$ is thus

$$
\omega \tilde{f}_{k}+J_{1}\left[h ; x_{k}\right]=g\left(x_{k}\right), \quad k=1,2, \ldots, n
$$

More explicitly,

$$
\omega \tilde{f}_{k}+\sum_{i=1}^{n}\left[\frac{8}{7} w_{n}\left(x_{k}, x_{i}\right)-\frac{1}{7} \epsilon_{k, i}^{(1)} w_{n / 2}\left(x_{k}, x_{i}\right)\right] \tilde{f}_{i}=g\left(x_{k}\right), \quad k=1,2, \ldots, n
$$

where

$$
\epsilon_{k, i}^{(1)}= \begin{cases}1 & \text { if } k-i \text { even } \\ 0 & \text { if } k-i \text { odd }\end{cases}
$$

By applying two extrapolations, we can remove the terms $\beta_{k}(t) h^{2 k+1}, k=$ 1,2 , from the asymptotic expansion of $I[h ; t]$. This time we choose $h=T / n$ for an integer $n$ that is divisible by 4 , and let $x_{i}=a+i h, i=0,1, \ldots, n$. Performing the two extrapolations, we obtain the Romberg-type quadrature rule

$$
\begin{aligned}
J_{2}[h ; t] & =\frac{32}{31} J_{1}[h ; t]-\frac{1}{31} J_{1}[2 h ; t] \\
& =\frac{256}{217} I[h ; t]-\frac{40}{217} I[2 h ; t]+\frac{1}{217} I[4 h ; t]
\end{aligned}
$$

as the new approximation to $I[t]$. We also have

$$
J_{2}[h ; t] \sim I[t]+\sum_{k=3}^{\infty} \frac{2^{3}-2^{2 k+1}}{7} \cdot \frac{2^{5}-2^{2 k+1}}{31} \beta_{k}(t) h^{2 k+1} \text { as } h \rightarrow 0,
$$


hence $J_{2}[h ; t]-I[t]=O\left(h^{7}\right)$ as $h \rightarrow 0$. The quadrature method for (4) based on $J_{2}[h ; t]$ is thus

$$
\omega \tilde{f}_{k}+J_{2}\left[h ; x_{k}\right]=g\left(x_{k}\right), \quad k=1,2, \ldots, n .
$$

More explicitly,

$$
\begin{aligned}
\omega \tilde{f}_{k}+\sum_{i=1}^{n}\left[\frac{256}{217} w_{n}(\right. & \left.x_{k}, x_{i}\right)-\frac{40}{217} \epsilon_{k, i}^{(1)} w_{n / 2}\left(x_{k}, x_{i}\right) \\
+ & \left.\frac{1}{217} \epsilon_{k, i}^{(2)} w_{n / 4}\left(x_{k}, x_{i}\right)\right] \tilde{f}_{i}=g\left(x_{k}\right), \quad k=1,2, \ldots, n,
\end{aligned}
$$

where $\epsilon_{k, i}^{(1)}$ are as before and

$$
\epsilon_{k, i}^{(2)}= \begin{cases}1 & \text { if } k-i \text { divisible by } 4 \\ 0 & \text { otherwise }\end{cases}
$$

For the development of Romberg-type formulas of all orders for all types of weak singularities, we refer the reader to Sidi and Israeli [7.

\section{References}

1. H.B. Keller. Numerical Methods for Two-Point Boundary Value Problems. Blaisdell, Waltahm, Mass., 1968.

2. J.A. Pennline. Improving convergence rate in the method of successive approximation. Math. Comp., 37:127-134, 1981.

3. J.A. Pennline. Constructive existence and uniqueness for some nonlinear two-point boundary value problems. J. Math. Anal. Appl., 96:584-598, 1983.

4. J.A. Pennline. Constructive existence and uniqueness for two-point boundary value problems with a linear gradient term. Appl. Math. Comp., 15:233-260, 1984.

5. A. Sidi. Comparison of some numerical quadrature formulas for weakly singular periodic Fredholm integral equations. Computing, 43:159-170, 1989.

6. A. Sidi. Practical Extrapolation Methods: Theory and Applications. Number 10 in Cambridge Monographs on Applied and Computational Mathematics. Cambridge University Press, Cambridge, 2003.

7. A. Sidi and M. Israeli. Quadrature methods for periodic singular and weakly singular Fredholm integral equations. J. Sci. Comput., 3:201-231, 1988. Originally appeared as Technical Report No. 384, Computer Science Dept., Technion-Israel Institute of Technology, (1985), and also as ICASE Report No. 86-50 (1986).

8. A. Sidi and J.A. Pennline. Improving the accuracy of quadrature method solutions of Fredholm integral equations that arise from nonlinear two-point boundary value problems. J. Integral Eqs. Appl., 11:103-139, 1999. 